\title{
Experimental studies of reinforced concrete shear walls retrofitted with slotted steel plates
}

\author{
B. Cheng ${ }^{1}$, X.J. Gao' ${ }^{2}$ M.J. Zhou ${ }^{3}$, Q. Zhang ${ }^{4}$ \\ 1,2,3,4 Department of Civil \& Transportation Engineering, Beijing University of Civil Engineering and \\ Architecture, China \\ achengbei@bucea.edu.cn, b2108140216008@stu.bucea.edu.cn
}

\begin{abstract}
Keywords: strengthening; shear wall; slotted steel plate; collapse performance
\end{abstract}
Abstract. Many concrete shear walls are always subjected to high gravitational loads and prone to brittle shear failure under the lateral seismic loading which probably leads to the collapse of the whole shear walls system. This paper aims to present a new method of using slit steel plates to retrofit reinforced concrete shear walls, and emphatically analyze the anti-collapse and anti-seismic performance of the retrofitted specimens. A total of 4 shear wall specimens are conducted under lateral cyclic loading tests. One is the original specimen and the others are reinforced with slotted steel plates. The experimental results show that the specimens with slotted steel pate can achieve better anti-collapse performances than that without opening of steel plate.

\section{Introduction}

Earthquake can cause huge casualties and property damage. Therefore, the seismic capacity of structures, especially the earthquake resistance ability, is the most important component of seismic disaster prevention capability in earthquake areas[1]. In 2007, Wang Xinjie[2] conducted a study on improving the seismic performance of low-rise reinforced concrete(RC) shear wall. It was pointed out that low-rise reinforced concrete shear wall was always been found in the bottom conversion layer for using more bottom large-space in recent years. And the failure mode of the low-rise RC shear wall is brittle shear failure with poor ductility and energy dissipation capacity. Meanwhile, the failure may even cause an overall collapse of the building under strong earthquake.

Matsui and Hitaka from Kyushu University introduced a steel plate shear wall with slits in 2000[3]. As the presence of vertical slits changes the stress state of the steel plates, bending and yielding of the steel plate segments between the slits can occur before overall buckling of the steel plates. This offers the shear wall good ductility and energy dissipation capability. Moreover, the shear wall's stiffness, bearing capacity, ductility, and energy dissipation capability can be adjusted by changing the parameters and forms of slits[4].

\section{Test Setup}

\section{Specimen design and loading method}

Four identical shear wall members with height-to-width ratio of 1: 1 are designed, identified as SW2, SW2-1, SW2-2 and SW2-3. Their specific dimensions and reinforcement forms are shown in Fig.1. Main materials used in the specimens included C40 concrete, Q235 steel plate, and HRB500 and HPB300 rebars. The specimen reinforcement scheme is shown in Tab.1. 

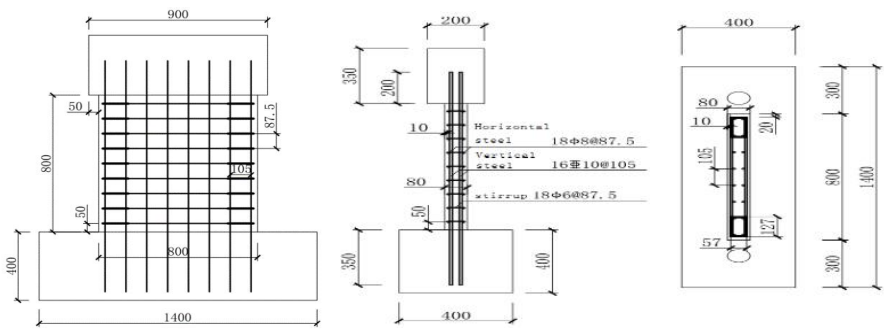

Tab.1 Strengthening scheme of specimens

\begin{tabular}{cc}
\hline Specimen & Reinforcement form \\
\hline SW2 & no steel plate reinforcement \\
SW2-1 & non-slit steel plate reinforcement \\
SW2-2 & single slit steel plate reinforcement \\
SW2-3 & double slit steel plate reinforcement \\
\hline
\end{tabular}

Fig.1 Reinforcement details of specimens

\section{Loading method}

A $500 \mathrm{kN}$ actuator provides horizontal reciprocating load, and a $1000 \mathrm{kN}$ jack provides vertical load. At the beginning of the test, an axial force of $489 \mathrm{kN}$ is applied, and the axial compression ratio remains 0.4 during the test. The horizontal load is loaded by the hydraulic servo loading system. After the vertical load reaches the specified value, the horizontal reciprocating load is applied by the loading system shown in Fig.2. And each level cycle is repeated twice. The test is stopped when the vertical load reaction force is significantly reduced (collapsed) or the specimen is damaged.

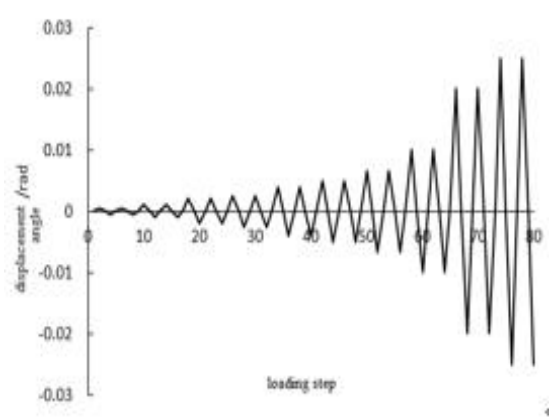

Fig.2 Loading sequence

\section{Test results analysis}

\section{Failure modes and steel deformation}

Fig.3(a) shows the failure modes of the four specimens. Diagonal cracks occurs in specimen SW2 and its bottom concrete is heavily damaged and falls off, which are typical characteristics of buckling failure. In specimen SW2-1, the steel plate without a slit only deforms slightly and the concrete at the bottom exhibits noticeable cracks, which are similar to the concrete failure mode of SW2. For specimen SW2-2, it can be seen in Fig.3(c) that both the left and right parts of the single-slit steel plate undergo significant deformation. In specimen SW2-3, the left segment of the steel plate deforms significantly, while the middle and right segments display slight deformation. It can be found that the deformation of the single-slit steel plate in specimen SW2-2 is obviously larger than that of the double-slit steel plate in SW2-3, while the deformation of the non-slit steel plate in SW2-1 is the smallest.

Fig.4 shows the vertical strain value at the same middle position of the three steel plates. It can also be found that specimen SW2-1 with the non-slit steel plate has the smallest deformation. And the deformation of the single-slit steel plate in specimen SW2-2 is obviously larger than that of the double-slit steel plates in SW2-3. Therefore, the changes of the number and position of the slits can make a different deformation between the retrofitted specimens.

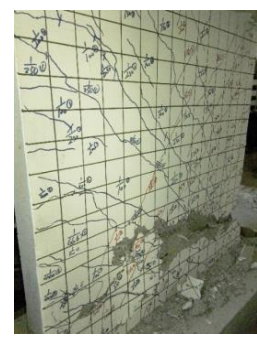

(a)SW2

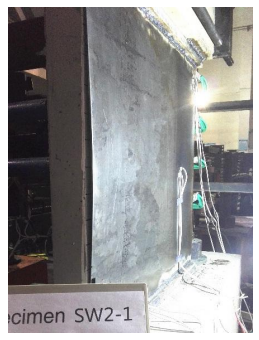

(b)SW2-1

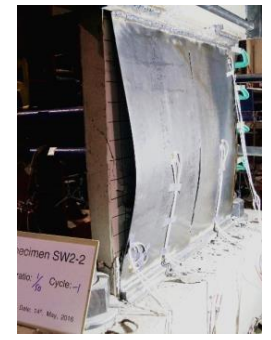

(c)SW2-2

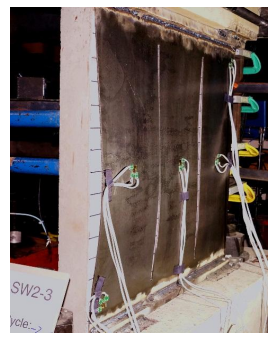

(d)SW2-3

Fig.3 Failure modes of specimen

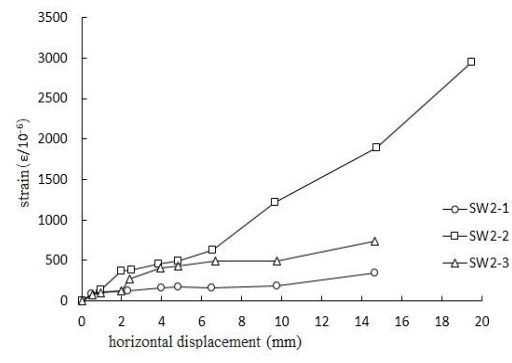

Fig.4 Comparison of steel plate strain 


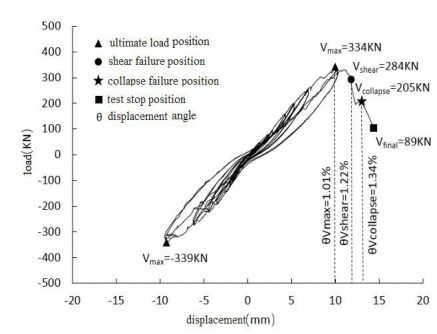

(a1)SW2 Horizontal force hysteresis curve

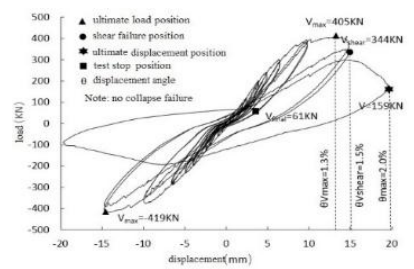

(c1)SW2-2 Horizontal force hysteresis curve

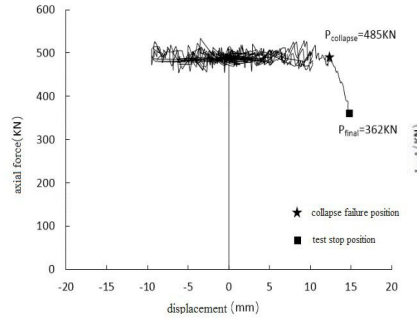

(a2)SW2 Axial force hysteresis curve

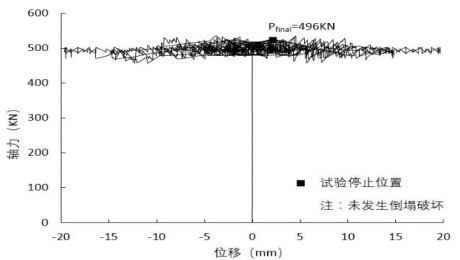

(c2)SW2-2 Axial force hysteresis curve

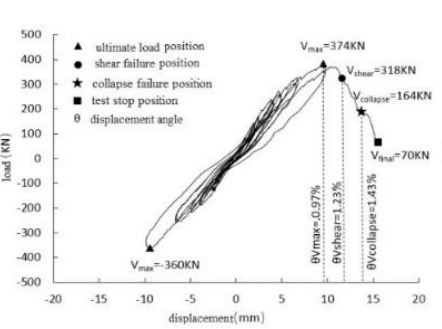

(b1)SW2-1 Horizontal force hysteresis curve

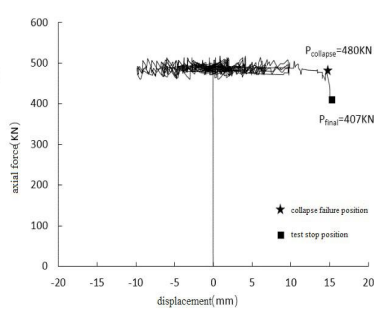

(b2)SW2-1 Axial force hysteresis curve

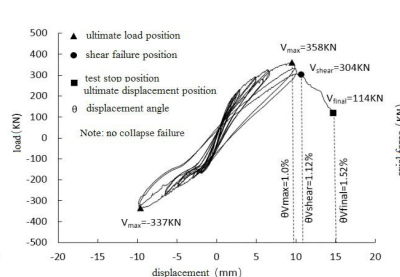

(d1)SW2-3 Horizontal force hysteresis curve

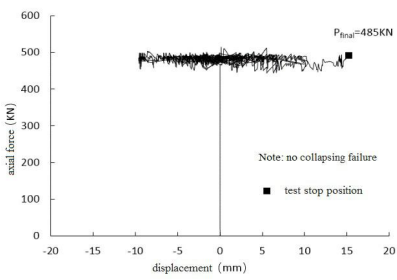

(d2)SW2-3 Axial force hysteresis curve

Fig.5 Hysteresis curves of Specimen

\section{Hysteresis curve}

Fig.5 shows the hysteresis curves of the four specimens and several key performance points. Fig.5(a2) reveals that the vertical load of SW2 is suddenly dropped, which means the specimen tends to collapse and cannot maintain the specific axial force. Fig.5(a1) shows the limit displacement angle of the collapse position $\left(V_{\text {collapse }}\right)$ is $1.34 \%$. The same situation occurs in specimen SW2-1 as shown in Fig.5(b2), and the corresponding limit displacement angle is $1.43 \%$. The steel plate without a slit also demonstrates low deformation capacity. Specimens SW2 and SW2-1 have similar failure characteristics, which indicates that the steel plate without a slit is ineffective in strengthening the collapse resistance capability of shear walls.

Fig.5(c1) reveals that specimen SW2-2 can behave more ductile, and the limit displacement angle is 2.0\%. Moreover, as shown in Fig.5(c2), specimen SW2-2 can withstand the specific vertical load without a collapsing failure. From Fig.5(d1), the limit displacement angle of SW2-3 is $1.52 \%$, without a collapse failure. Through above comparisons, specimens strengthened with slit steel plates, especially SW2-2 whose hysteresis loop covers a relatively large area and is plump in shape, have relatively high energy dissipation and collapse resistance capability.

\section{Ductility coefficient and energy dissipation}

The skeleton curves of each specimen are shown in Fig.6.

The coefficient $\theta_{y}$ and $\theta_{u}$ respectively represent the yield displacement angle and the limit displacement angle. It can be seen from Tab.2 that the ductility coefficient $(\mu)$ of the specimens SW2 and SW2-1 are similar, because the non-slit steel plate without a sufficient deformation has no contribution to ductility. The slit steel plates used in SW2-2 and SW2-3 are easy to deform so that their ductility capacity is increased compared with the previous two specimens.

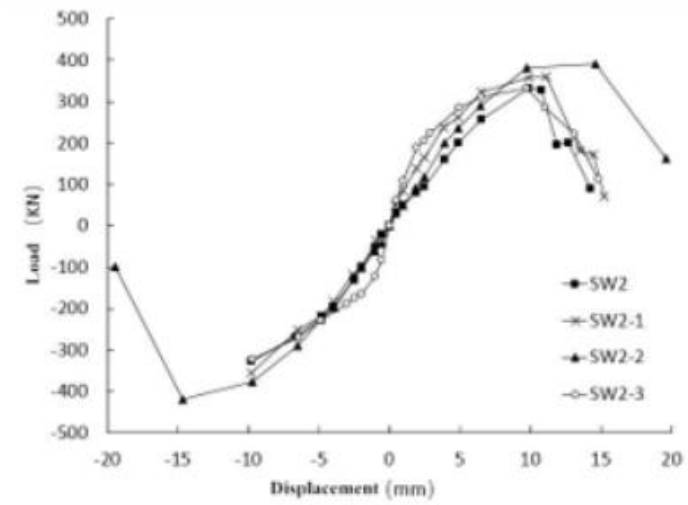

Fig.6 Comparison of skeleton curves

Energy dissipation coefficient $(E)$ and Equivalent viscous damping $\operatorname{coefficient}\left(\xi_{\mathrm{eq}}\right)$ are 
calculated (as shown in Tab.2) to judge the energy dissipation ability of the specimens. A specimen with a higher dissipation factor can dissipate more seismic energy and have a better seismic performance.

Tab.2 Ductility and energy dissipation coefficient

\begin{tabular}{cccccc}
\hline Specimen & $\theta_{y}(\%)$ & $\theta_{u}(\%)$ & $\mu$ & $E$ & $\xi_{\text {eq }}$ \\
\hline SW2 & 1.02 & 1.49 & 1.46 & 0.42 & 0.067 \\
SW2-1 & 1.01 & 1.50 & 1.48 & 0.46 & 0.073 \\
SW2-2 & 1.02 & 1.83 & 1.79 & 1.71 & 0.272 \\
SW2-3 & 0.85 & 1.37 & 1.62 & 0.59 & 0.094 \\
\hline
\end{tabular}

The results above demonstrate that the use of steel plates improves the energy dissipation capability of the shear wall specimens. The energy dissipation of specimens SW2-2 and SW2-3 is higher than that of SW2 and SW2-1. And specimen SW2-2 with single-slit steel plate differed greatly from SW2-3 with the double-slit steel plate in energy dissipation capability. This may due to the reason that the energy dissipation in SW2-1 with the non-slit steel plate mainly relies on the inclined tension field emerged after the steel yield. Besides, specimen with slit steel plate relies on the bending deformation of the columns between slits to dissipate energy. Since the middle of the top beam has the minimum vertical displacement, the middle of the steel plate with the double-slit is hardly deformed. Therefore, the single-slit steel plate in specimen SW2-2 has a better deformation capacity than the double-slit steel plate in SW2-3.

\section{Conclusion}

(1) It is found that the use of slit steel plates can increase the deformation capacity and energy dissipation capability of existing shear walls with small span to height ratios, and thereby improve their seismic performance.

(2) Positions and numbers of slits have a great influence on deformation capacity of a slit steel plate. And the collapse resistance capability is associated with the deformation capacity of steel plate.

\section{Acknowledgements}

The work described in this paper has been fully supported by the National Natural Science Foundation (Project No.51208023), Beijing Natural Science Foundation (Project No.8162014) and Beijing advanced innovation center for future urban design (Project No. UDC2016030200).

\section{References}

[1] L.P. Ye, Z. Qu. The design and methods of improving the seismic collapse resistance of building structures (in Chinese). Journal of Building Structures, p. 42-50. (2008)

[2] X.J. Wang, Wan-Lin Cao. Research on improvement of seismic performance of low-rise RC shear wall (in Chinese). World Earthquake Engineering, p. 61-66. (2007)

[3] T. Hitaka, C. Matsui. Experimental study on steel shear wall with slits. Journal of Structural Engineering, p. 586-595. (2003)

[4] X.B. Ma. Seismic behavior of steel plate shear walls and composite shear walls with two-side connections (in Chinese). HARBIN Institute of Technology. (2009) 